\title{
Case Study in Refractory Non-Hodgkin's Lymphoma: Successful Treatment with Plerixafor
}

\author{
Hamdy Abdel Azim ${ }^{a}$ Sherif Ahmed Bahr ${ }^{b}$ \\ Mohammed Adel Koura ${ }^{a}$ Heba Gad $^{\mathrm{e}}$ Mohamed Farouk $^{c}$ \\ Ahmed Morsy $^{a}$ Ibrahim Iskander ${ }^{a} \quad$ Ahmed Hammad $^{a}$ \\ Mohammed Fat'hy ${ }^{d}$ Hossam Attia $^{d}$ Karim Sadek $^{a}$ \\ Departments of ${ }^{\mathrm{a}} \mathrm{Clinical}$ Oncology, ${ }^{\mathrm{b}}$ Oncology and Hematology, ${ }^{\mathrm{C}} \mathrm{Clinical}$ \\ Hematology, ${ }^{\mathrm{d}}$ Medical Oncology, and ${ }^{\mathrm{e}} \mathrm{Clinical}$ Pathology, BMT and Clinical \\ Oncology Unit, Faculty of Medicine, Cairo University, Cairo, Egypt
}

\section{Key Words}

Diffuse large cell lymphoma · Non-Hodgkin's lymphoma - Chemotherapy · Plerixafor · Granulocyte colony-stimulating factor $\cdot$ Stem cell mobilization

\begin{abstract}
The present case study describes our experience in treating a young woman diagnosed with a relapsing case of diffuse large cell lymphoma, who was heavily pre-treated with chemotherapy and radiotherapy. Our only chance to improve her survival was by using highdose chemotherapy, followed by peripheral stem cell rescue. Unfortunately, in this patient, collecting sufficient stem cells for bone marrow transplantation proved to be very difficult since she had already been heavily treated with chemotherapy and radiotherapy. Currently, granulocyte colony-stimulating factor (G-CSF) alone or G-CSF plus chemotherapy are the most commonly used treatments for stem cell mobilization. However, $5-30 \%$ of patients do not respond to these agents. Plerixafor is a new hematopoietic stem cell-mobilizing drug that antagonizes the binding of chemokine stromal cell-derived factor- $1 \alpha$ to CXC chemokine receptor 4. It is indicated in combination with G-CSF to mobilize hematopoietic stem cells to the peripheral blood for collection and subsequent autologous transplantation in patients with non-Hodgkin's lymphoma and multiple myeloma [Kessans et al.: Pharmacotherapy 2010;30:485-492; Jantunen: Expert Opin Biol Ther 2011;11:1241-1248]. Based on our findings, we consider plerixafor to be a very efficient and practical solution to mobilize and collect stem cells among all patients in such a situation, enabling us to proceed to autologous bone marrow transplantation and peripheral stem cell rescue in order to improve the patients' overall survival.
\end{abstract}




\section{Case Report}

This report follows the case of a 20 -year-old female patient who was diagnosed in 2008 with refractory non-Hodgkin's lymphoma (diffuse large cell lymphoma, CD20 positivity). Her disease was at stage IIISB when it was initially misdiagnosed as Hodgkin's lymphoma. As a result, she received 4 cycles of classic ABVD regimen [adriamycin (doxorubicin), bleomycin, vinblastine and dacarbazine]; however, no response could be observed. Her treatment was then changed to the classic ICE regimen (ifosfamide administered as an infusion with bolus carboplatin, and etoposide). She underwent 4 cycles of this therapy, with major partial remission. This was followed by classic mantle-field radiotherapy (4,500 cGy/25 fractions). Immediately after completion of the radiotherapy course, the patient had a major relapse in the form of abdominal lymphadenopathy, especially in the porta hepatis lymph nodes, leading to obstructive jaundice. The patient underwent a CT-guided biopsy of a mediastinal mass, together with immunophenotyping, which revealed diffuse large B-cell lymphoma with positive CD20 staining. Therefore, we considered that her regimen should contain rituximab in order to give her the best chance of an improved response and longer overall survival. The other components of the regimen were chemotherapeutic agents active against lymphoma and, in order to avoid drug resistance, not previously used in this patient. Thus, the following treatment was initiated: rituximab $375 \mathrm{mg} / \mathrm{m}^{2}$ on day 0 ; cyclophosphamide $250 \mathrm{mg} / \mathrm{m}^{2}, 12 \mathrm{~h}$, on days $1-3$; cisplatin $25 \mathrm{mg} / \mathrm{m}^{2}$ on days 1-4; methylprednisolone $250 \mathrm{mg}$ /day on days 1-4, and etoposide $70 \mathrm{mg} / \mathrm{m}^{2}$ on days 1-4 with granulocyte colony-stimulating factor (G-CSF) support.

The patient attained major partial remission after the second cycle of the treatment regimen described above. Therefore, mobilization of peripheral blood stem cells (PBSCs) was attempted by the third cycle. The regimen described was given, followed by G-CSF on a daily basis at $10 \mu \mathrm{g} / \mathrm{kg} / \mathrm{day}$, starting from day 9. Daily measurements of peripheral blood CD34 cells were performed. However, the peripheral CD34-positive cells never exceeded 3 cells/ $\mu$ l until day 19, indicating that the mobilization had failed.

After 2 weeks of treatment, another trial of mobilization with the same regimen, but without rituximab, was carried out using the same doses of chemotherapy, followed by G-CSF at $10 \mu \mathrm{g} / \mathrm{kg}$ on day 9. Daily measurements of peripheral blood CD34 cells were taken until day 21 . However, again the peripheral CD34-positive cells did not exceed 3 cells/ $\mu$ l.

Since the patient had been heavily pre-treated with chemotherapy and radiotherapy, with all attempts of mobilization by means of chemotherapy and G-CSF having failed, we tried to mobilize the patient's PBSCs a third time using the immune stimulant plerixafor and G-CSF. Therefore, 2 weeks after completing the treatment, we initiated the following treatment regimen: G-CSF $10 \mu \mathrm{g} / \mathrm{kg}$ s.c. administered daily in the morning for 4 days (days 1-4); on the evening of day 4 , the patient received plerixafor at a dose of $0.24 \mathrm{mg} / \mathrm{kg}$ s.c.; the time for the evening plerixafor was adjusted to exactly $10-$ $11 \mathrm{~h}$ before pheresis and 3-5 $\mathrm{h}$ after the morning G-CSF.

This regimen was repeated for 2 consecutive days, with peripheral blood CD34-positive cells being 57 and 45 cells/ $\mu$ l on the first and second day, respectively. The number of CD34-positive cells collected after the first pheresis was $2.7 \times 10^{6}$ cells $/ \mathrm{kg}$ body weight, and $1.2 \times 10^{6}$ cells $/ \mathrm{kg}$ body weight after the second pheresis.

\section{Discussion}

There were many risk factors that contributed to the poor mobilization in this patient. These included more than 10 cycles of chemotherapy, use of a platinum-based regimen, dorsal vertebrae radiotherapy, and two failed mobilization attempts using chemotherapy with G-CSF.

Based on the results of the third trial, plerixafor proved to be a very effective and practical drug for mobilization of PBSCs in bone marrow-depleted patients, especially if the peak response of plerixafor can be adjusted to the peak serum level of G-CSF (between 10 and $14 \mathrm{~h}$ before) and blood pheresis (between 3 and $5 \mathrm{~h}$ after). 


\section{Conclusion}

The results of this case study suggest that mobilization of heavily pre-treated patients that failed following chemotherapy and G-CSF can be attempted using plerixafor and G-CSF. This approach may offer patients potentially better chances for mobilization, followed by high-dose chemotherapy and peripheral stem cell rescue. We suggest that the method of adjusting the peak serum level of plerixafor to the peak serum level of G-CSF and the blood pheresis could lead to greater efficacy of plerixafor and stem cell yield. These adjustments could be used in future mobilizations and compared in a retrospective manner with conventional mobilization by plerixafor. Further investigations in randomized clinical trials are therefore warranted.

\section{References}

1 Kessans MR, Gatesman ML, Kockler DR: Plerixafor: a peripheral blood stem cell mobilizer. Pharmacotherapy 2010;30:485-492.

-2 Jantunen E: Novel strategies for blood stem cell mobilization: special focus on plerixafor. Expert Opin Biol Ther 2011;11:1241-1248. 